\title{
Um Sistema de Diagnóstico de Leucemia utilizando CNN's Pré-treinadas e um Comitê de Classificadores
}

\author{
Luis H. S. Vogado, Rodrigo M. S. Veras, Alan R. Andrade, Luís G. T. Santos, \\ Kelson R. T. Aires, Vinicius P. Machado \\ Departamento de Computação, \\ Universidade Federal do Piauí, Teresina-PI \\ lhvogado@gmail.com, rveras@ufpi.edu.br, alanribeiroandrade@gmail.com, \\ luisguilherme.ufpi@gmail.com, \{kelson, vinicius\}@ufpi.edu.br
}

\begin{abstract}
Leukemia is among the diseases that afflict more young people and adults, thus causing an early death. To assist the experts in diagnosing this disease, there are computer-aid systems. These systems prevent diagnosis from being affected by variables such as experience and the tiredness of the specialist, collaborating with a prescription medication and avoiding inappropriate treatment. In this work, a new methodology for the creation of a system of diagnosis of leukemia with the use of CNN's, PCA and an ensemble of classifiers. The ALL-IDBI database was used in conducting the experiments and obtained 98.14\% accuracy, overlapping the results presented in the literature.
\end{abstract}

Resumo. A leucemia está entre as doenças que mais afligem os jovens e adultos, causando assim uma morte precoce. Para auxiliar os especialistas no diagnóstico dessa doença, existem sistemas de auxílio por computador. Estes evitam que os diagnósticos sofram com variáveis como experiência e o cansaço do especialista, colaborando com uma prescrição de medicamentos mais adequada e evitando um tratamento inadequado. Neste trabalho, é apresentada uma nova metodologia para a criação de um sistema de diagnóstico da leucemia com o uso de CNN's, PCA e um comitê de classificadores. A base de dados ALL-IDBI foi utilizada na realização dos experimentos e obtivemos $98,14 \%$ de acurácia, sobrepondo os resultados apresentados na literatura.

\section{Introdução}

O diagnóstico é um importante processo realizado por médicos que consiste em determinar a presença ou não de doenças baseando-se em um conjunto de dados. Estes dados são primordiais para a identificação de doenças e podem ser compostos por sinais, sintomas, imagens, exames entre outros. Um diagnóstico errôneo, causado por um exame mal sucedido, pode ocasionar efeitos colaterais ao paciente, devido a uma possível prescrição de medicamentos inadequados para o tratamento. Para auxiliar os especialistas nesta etapa crucial, existem sistemas de baixo custo que analisam e processam os dados, provendo um auxílio ao diagnóstico.

Ao longo dos anos, foram propostos múltiplos sistemas de auxílio médico. Doenças como glaucoma [Chen et al. 2015], câncer de pele [Kawahara and Hamarneh 2016], 
câncer de mama [Wang et al. 2016] e leucemia [Madhukar et al. 2014] foram abordadas nesses sistemas. O diagnóstico precoce dessas doenças é fundamental para o seu tratamento. Este por sua vez pode ser dispendioso e complexo, que mesmo em alguns casos, não curando o paciente, prolonga a sua vida.

Dentre as doenças abordadas nos sistemas de auxílio por computador, a leucemia é a que possui maior número de vítimas fatais entre adolescentes e crianças, sendo que o risco de desenvolvê-la é maior em crianças de até 5 anos. A leucemia é um câncer que se origina na medula óssea e é caracterizado pela proliferação anormal de glóbulos brancos (Figura 1). O diagnóstico da leucemia pode ser feito por meio de várias análises e exames, dentre eles o exame físico, teste de sangue, hemograma, mielograma, punção lombar e biópsia na medula óssea. A realização do exame que utiliza a análise microscópica é a maneira mais econômica para a triagem inicial de pacientes com leucemia. Esse tipo de exame é feito manualmente o que acaba gerando cansaço e fadiga no operador, justificando a necessidade de um sistema de auxílio.
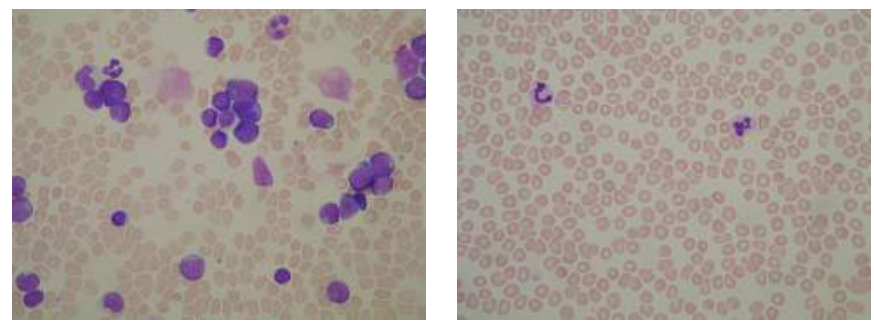

\section{Figura 1. Exemplos de lâminas de sangue com leucemia(esquerda) e sem(direita). Ambas pertencentes a base ALL-IDB 1.}

Parte desses sistemas são desenvolvidos com a utilização de técnicas de processamento de imagens e inteligência computacional e geralmente possuem etapas de préprocessamento, segmentação, extração de características e classificação. As etapas que melhor definem o diagnóstico realizado por esses sistemas são a extração de características e a classificação. No entanto, para conseguir mais robustez, essas etapas acabam dependendo de suas predecessoras, ou seja, uma boa segmentação pode prover uma boa extração e consequentemente uma boa classificação.

Há alguns anos, pesquisadores que trabalham com inteligência artificial utilizam aprendizado profundo através das Redes Neurais Convolucionais (CNNs - Convolutional Neural Networks), possibilitando a criação de poderosos sistemas computacionais de auxílio médico. O poder dessas redes é aplicado em diversas áreas, como o processamento de sinais, linguagem natural, imagens e vídeo. Contudo, são necessárias máquinas com grande capacidade de processamento e uma enorme quantidade de dados para treiná-las e adequá-las a tarefa desejada.

A abordagem proposta neste trabalho descreve um sistema de diagnóstico da leucemia que não necessita da etapa de segmentação (uma etapa comumente apresentada em trabalhos no estado da arte). Este trabalho consiste em extrair diretamente as características da imagem com um descritor baseado em aprendizado profundo e classificá-las. Na 
etapa de extração, utilizamos as CNN's pré-treinadas AlexNet, VggNet e CaffeNet. Para a classificação, propomos um comitê com três classificadores, o Support Vector Machine (SVM), o Multilayer Perceptron (MLP) e o Random Forest (RF). Procuramos avaliar a performance do sistema em uma base com lâminas de sangue contendo diversos núcleos. Os resultados obtidos são comparados com outros sistemas existentes no estado da arte.

O restante do texto está dividido da seguinte forma: apresentamos os trabalhos relacionados na Seção 2, e a metodologia proposta na Seção 3. Na Seção 4 descrevemos as bases de imagens utilizadas nos testes e discutimos os resultados obtidos. Finalmente, as conclusões e perspectivas para trabalhos futuros na Seção 5.

\section{Trabalhos Relacionados}

Diversos sistemas utilizados para auxiliar no diagnóstico da leucemia foram propostos ao longo do tempo. Dentre estes, alguns apresentaram soluções para a classificação dos dois tipos de leucemia mais comuns, são elas: a leucemia mieloide aguda (LMA) e a leucemia linfóide aguda (LLA), utilizando imagens de lâminas de sangue.

No trabalho proposto por Madhukar et al.[Madhukar et al. 2012], os autores propuseram a criação de um sistema para detectar LLA utilizando uma base de dados com apenas um núcleo por imagem. O pré-processamento aplicado consiste na conversão da imagem no sistema de cor RGB para o $\mathrm{L}^{*} \mathrm{a}^{*} \mathrm{~b}$, um sistema que é geralmente empregado no agrupamento de imagens. Na etapa de segmentação, o algoritmo não-supervisionado $K$-means é aplicado nos canais *a e *b da imagem, sendo o número de grupos igual a três. Na extração de características, um conjunto de descritores foi utilizado, sendo eles: características de forma, Gray Level Co-occurrence Matrix (GLCM) e dimensão Fractal. Na realização dos testes, foram utilizadas 98 imagens de lâminas de sangue da base ALLIDB1 [Labati et al. 2011]. A classificação foi realizada com o Support Vector Machine e três técnicas para a validação cruzada dos dados: $k$-fold, Hould-Out e Leave-One-Out. Com os dados analisados, os autores concluíram que a técnica que obteve a melhor acurácia foi o Leave-One-Out com 93,50\%.

Vincent et al. [Vincent et al. 2015] propuseram a criação de um sistema que utiliza redes neurais como classificador. A execução deste método proposto consiste em um pré-processamento inicial com agrupamento baseado no sistema de cor $\mathrm{L}^{*} \mathrm{a} \mathrm{a}^{*} \mathrm{~b}$ e filtros morfológicos. A etapa de classificação é composta por dois passos, o primeiro é a extração de características, sendo o vetor formado por um conjunto de cinco tipos de características, são elas: textura (GLCM com a entropia), contraste, correlação, energia e o Hausdorff Dimension. O Principal Component Analysis (PCA) é aplicado na busca das melhores características e então o vetor é classificado pela rede neural. O resultado provido pelo classificador é a diferenciação das células entre normais e anormais. Estas que servirão de entrada para o segundo passo, a classificação foi realizada com mais um conjunto de características, que agora envolvem a geometria das células. As cinco características escolhidas neste passos foram: área da célula, do núcleo e do citoplasma, a relação núcleo/citoplasma e núcleo/célula. O PCA foi aplicado novamente com o mesmo propósito de reduzir as características do vetor. A base ALL-IDB1 foi utilizada nos testes e os resultados obtidos foram de 97,70\% de acurácia para 100 imagens.

No trabalho de Patel e Mishra [Patel and Mishra 2015], os autores apresentaram um sistema automático para a detecção de leucemia em imagens microscópicas de san- 
gue. O trabalho proposto contém as fases de pré-processamento, segmentação, extração de características e classificação. Na primeira fase, filtros foram utilizados para remover possíveis ruídos na imagem, para assim facilitar a segmentação da mesma. Os autores, diferente de outros trabalhos do estado da arte, não fazem a mudança no sistema de cor, permanecendo assim com o RGB. Na etapa de segmentação, a imagem é convertida para tons de cinza e para agrupar, o K-means e o algoritmo de Zack [Zack et al. 1977] são aplicados. Na extração, as características de cor, geometria, textura e estatísticas foram utilizadas. A base de imagens utilizada, assim como nos trabalhos apresentados anteriormente, é a ALL-IDB1, no entanto, apenas 27 imagens foram utilizadas nos testes. Com o SVM, foram obtidos $93,57 \%$ de acurácia.

O sistema proposto por Agaian et al. [Agaian et al. 2016] apresentou uma abordagem para a classificação de imagens de sangue com múltiplos núcleos. Os autores aplicaram a conversão dos sistemas de cor do RGB para o L*a*b e realizaram o agrupamento com o K-means. As características selecionadas foram as de forma, cor, GLCM, Haar wavelet, dimensão Fractal e o SVM foi utilizado como classificador. Os autores enfatizaram que todas as taxas de acerto apresentadas no trabalho estão acima de $94 \%$ de acurácia. A base ALL-IDB1 é utilizada com 98 imagens.

Como foi apresentado nessa Seção, muitos métodos do estado da arte utilizam descritores semelhantes para realizar a distinção dos lâminas de sangue. No entanto, em pesquisas mais recentes, os autores estão utilizando técnicas de Deep Learning como descritores [Castelluccio et al. 2015] ou classificadores [Wang et al. 2016, Kumar et al. 2016], proporcionando a utilização de poderosas redes nesses sistemas de auxílio. Observamos que nos trabalhos mais atuais, essas técnicas estão sendo empregadas nos problemas de diagnóstico, mas não encontram-se trabalhos com leucemia. No nosso trabalho, propomos a utilização dessas técnicas como descritores, procurando assim avaliar a imagem como um todo, levando em conta todos os elementos pertencentes a ela, descartando a etapa de segmentação do núcleo.

\section{Método Proposto}

O método proposto neste trabalho tem como objetivo diagnosticar a leucemia em imagens de sangue. Seguindo o fluxograma apresentado na Figura 2, observa-se que a entrada do sistema é uma imagem sem nenhum pré-processamento ou segmentação, diferente dos métodos propostos no estado da arte. A partir da imagem de entrada, as CNN's são utilizadas para descrevê-la, e os vetores são concatenados e reduzidos. Para a etapa de classificação, um comitê é proposto neste trabalho, trazendo mais confiabilidade aos resultados e classificando assim as imagens em doentes ou não. Estas etapas são melhor descritas no decorrer do texto, justificando a utilização de cada componente no fluxograma geral da abordagem.

\subsection{Extração de características utilizando CNN's}

O processo de extração de características consiste na representação de um conjunto redundante de dados, por meio de características únicas que o façam se diferenciar de outros conjuntos. Existem inúmeras características que podem ser extraídas, dentre elas, por exemplo: cor, forma e textura. Dentre os diversos descritores propostos no estado da arte, a utilização de CNN's neste processo de extração vem ganhando destaque. 


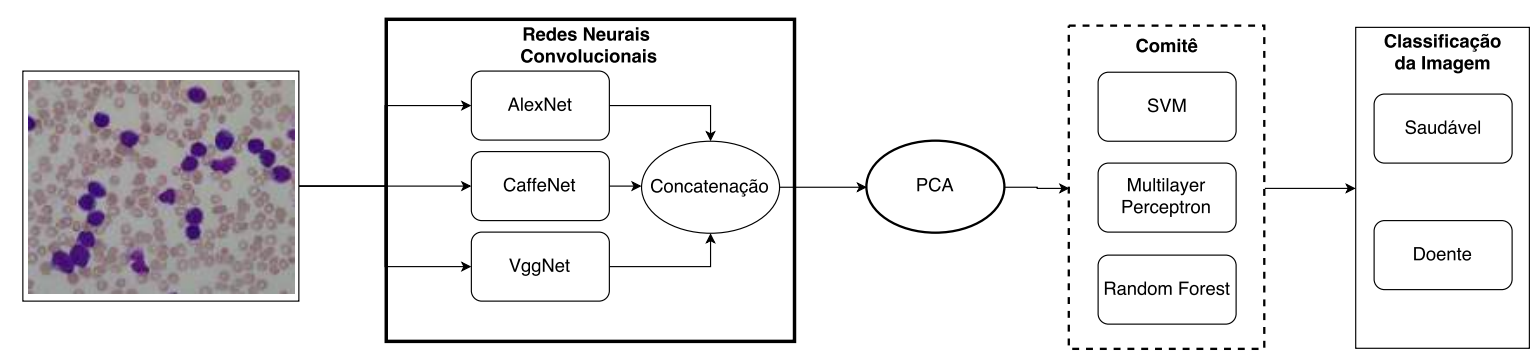

Figura 2. Fluxograma do método proposto.

No estado da arte, os autores apresentam duas formas de realizar o processo de aprendizagem de uma base de imagens com CNN's: a primeira é a forma usual, realizando o treinamento com um grande conjunto de dados, a segunda forma é a transferência de aprendizado, utilizando redes pré-treinadas.

No nosso trabalho, utilizamos a técnica de transferência de aprendizado [Shin et al. 2016] onde a CNN já é treinada em uma grande base natural de imagens fazendo com que assimile características genéricas, facilitando a sua aplicabilidade em pequenas bases de dados. Essa técnica pode ser utilizada em diversos tipos de tarefas, como por exemplo na extração de características de imagens de faces, objetos e doenças. O sucesso ou não dos resultados dependerá da similaridade da base utilizada para se extrair as características com a base de treinamento.

Duas formas de transferências são apresentadas no trabalho de Castellucio et al. [Castelluccio et al. 2015], a primeira consiste no fine-tune da rede, onde sua estrutura é modificada, congelando-se as camadas de alto nível. A segunda é a extração da penúltima camada totalmente conectada da rede, obtida a partir da imagem de entrada da rede e utiliza-se outro classificador no processo de classificação. Esta sendo a forma de transferência utilizada neste trabalho, como pode ser observado no fluxograma da Figura 2.

As CNN's que utilizamos são pré-treinadas na base natural de imagens ImageNet ${ }^{1}$. Três arquiteturas propostas recentemente foram utilizadas na construção da abordagem, são elas:

AlexNet: É uma arquitetura desenvolvida para a competição LSVRC-2010 e assim realizar o treinamento e a classificação da base de imagens ImageNet [Krizhevsky et al. 2012]. Compreende oito camadas que precisam ser treinadas, cinco camadas convolucionais seguidas de três camadas totalmente conectadas, bem como camadas de pooling.

CaffeNet: A arquitetura CaffeNet foi devenvolvida no Berkeley Vision and Learning Center (BVLC), sendo considerada uma das mais populares no aprendizado profundo [Jia et al. 2014]. Compreende 5 camadas convolucionais, cada uma seguida por uma camada de pooling, e 3 camadas totalmente conectadas.

VGGNet: A última das arquiteturas utilizadas na abordagem é a VGGNet, proposta em 2014 por [Chatfield et al. 2014], ela foi projetada para aumentar a profundidade das redes. Sendo considerada a mais difícil de se treinar, a rede requer uma maior me-

\footnotetext{
${ }^{1}$ Disponível em http://www.vlfeat.org/matconvnet/
} 
mória e tempo de execução do que a AlexNet. A rede contém 8 camadas de aprendizado, sendo 5 convolucionais e 3 totalmente conectadas.

Esses três modelos possuem arquiteturas semelhantes e a principal diferença entre essas é o tamanho dos filtros utilizados nas camadas convolucionais e na quantidade de neurônios das camadas totalmente conectadas A quantidade de elementos da camada retirada da rede é de 4096, sendo este o tamanho do vetor inicial. Com a grande quantidade de características, fez-se necessária a utilização de uma redução ou de uma seleção desses atributos.

\subsection{Seleção de características}

O PCA é uma técnica para a redução de dimensionalidade em um vetor de características. Foi desenvolvida em 1991 e tem como principal característica, reduzir um grande conjunto de dados procurando manter a variação presente neste conjunto, ou seja, nenhuma redundância de dados é encontrada. Esta redução é conseguida através da subtração da média de cada linha da matriz de características e o resultado, subtraímos de cada elemento da linha. A partir do conjunto de dados, a matriz de covariância é retirada e seus autovalores e autovetores são calculados, projetando os dados originais em um vetor de características de menor dimensão.

A aplicação do PCA é feita em diversos problemas, dentre eles, temos o reconhecimento facial, verificação de passaportes, registros médicos, etc. Em [Kumar et al. 2016], os autores utilizam o PCA com uma taxa de $90 \%$ na variação dos dados. Optamos por utilizá-lo no sistema apresentado neste trabalho com a mesma taxa de variação.

\subsection{Comitê}

A utilização de comitês no aprendizado de máquina é crescente há alguns anos. Formado por diversos classificadores, essa técnica de classificação é considerada mais eficiente que a utilização de apenas um classificador, pois proporciona uma maior confiabilidade nos resultados. Trabalhos apresentados na literatura ([Kumar et al. 2016, Mohapatra et al. 2014]) difundem o uso desta técnica em sistemas de auxílio por computador .

A escolha de diferentes classificadores, com propriedades distintas e sua combinação, corroboram com a eficiência do comitê. No nosso trabalho, foram selecionados três classificadores, baseados em testes e em trabalhos da literatura, são eles: Support Vector Machine (SVM), Multilayer Perceptron (MLP) e Random Forest (RF).

Existem diferentes formas de se combinar classificadores, geralmente usa-se a saída individual de cada um neste processo, isso minimiza a ocorrência de decisões errôneas. Cada imagem é classificada como doente ou não por cada classificador, a partir do resultado individual das imagens da base, é possível fazer a combinação dos resultados. Um dos meios mais utilizados na combinação é o voto majoritário, ele avalia a saída de cada classificador e se a maioria classificar a imagem como uma das classes, então esta será a sua classificação final. Além do voto majoritário, existem outras regras como o voto majoritário ponderado, contagem de borda, média e produto das probabilidades e a mediana [Jr. 2011].

No nosso trabalho, utilizamos a regra do voto majoritário e para realizar os treinamentos e testes do conjunto de dados, optamos pela utilização da validação cruzada 
$k$-fold, sendo o valor de $k$ igual a 10 . Também são utilizadas quatro métricas disponíveis na literatura, são elas: precisão, sensibilidade, acurácia, recall e o Índice Kappa. A implementação da etapa de extração de características do método foi feita no MATLAB, enquanto a etapa de redução e classificação das características foram feitas com a ferramenta WEKA.

\section{Experimentos}

\subsection{Base de Imagens}

Vários autores testaram seus sistemas com apenas uma base de dados, ou então nas suas próprias bases. No entanto, um dos desafios no processamento de imagens de sangue é a habilidade de diagnosticar a doença mesmo em bases com características distintas.

Proposta em [Labati et al. 2011], a ALL-IDB é uma base que contém duas versões distintas, denominadas de ALL-IDB1 e ALL-IDB2. Todas as imagens possuem a resolução nativa de $2592 \times 1994$ pixels, e a sua captura foi feita com uma câmera PowerShot G5.

A ALL-IDB 1 é composta por 108 imagens (59 saudáveis e 49 doentes) de lâminas de sangue, que possuem múltiplos leucócitos por imagem. Os linfoblastos desse conjunto foram demarcados por especialistas e são ao todo 510 elementos. Já a ALL-IDB2 possui 260 imagens e cada uma contém pelo menos um leucócito ou linfoblasto. Essa versão foi criada para testes em sistemas de classificação, enquanto a primeira tem como objetivo, testes com segmentação. Neste trabalho utilizamos a ALL-IDB1, considerando as imagens com todos os leucócitos.

\subsection{Resultados e Discussão}

Para validarmos o sistema proposto, foram realizadas diversas comparações, sendo elas, com a saída de cada arquitetura, com uma variação do método proposto acrescentando uma etapa de segmentação e com outros sistemas propostos no estado da arte. Nesta última comparação, escolhemos o método proposto por [Vogado et al. 2016]. Neste trabalho, os autores apresentaram um método robusto, com testes realizados em bases com características distintas e que em seus resultados, demonstraram superioridade diante de outros trabalhos do estado da arte.

Na Tabela 1 podemos observar os resultados da abordagem proposta comparados com as saídas de cada arquitetura. Nota-se que a concatenação das redes obteve uma melhor taxa de acerto se comparada com os resultados das arquiteturas separadas. Isso se deve ao fato de que a saída de cada rede possui um conjunto de dados que as caracterizam de uma forma única. O motivo por trás da concatenação dessas características foi para aproveitá-las de forma, para assim melhor representar os dados da imagem. No entanto, ao concatenar, o tamanho do vetor de atributos é multiplicado por três. Sendo assim, foi realizada a redução do vetor de características e consequentemente eliminando os atributos menos relevantes. Podemos observar que a saída das arquiteturas separadas também proporcionam um excelente índice Kappa e uma alta taxa de precisão e acurácia, destacando-se os modelos AlexNet e VggNet. Sendo que esta última atingiu taxas equivalentes a saída concatenada, sendo superada apenas no índice Kappa.

Outra comparação observada na mesma tabela, é entre a abordagem com e sem segmentação, onde esta última alcançou resultados superiores. Acreditamos que isso se 
Tabela 1. Resultados do desempenho das arquiteturas na base ALL-IDB 1 com e sem segmentação.

\begin{tabular}{ccccc}
\hline & $\mathbf{P}(\%)$ & $\mathbf{R}(\%)$ & $\mathbf{A}(\%)$ & $\mathbf{K}$ \\
\hline Com segmentação & & & & \\
\hline AlexNet & 96,40 & 96,30 & 96,29 & 0,9255 \\
CaffeNet & 94,40 & 94,40 & 94,44 & 0,8879 \\
VggNet & 96,40 & 96,30 & 96,29 & 0,9255 \\
AlexNet + CaffeNet + VggNet & $\mathbf{9 7 , 4 0}$ & $\mathbf{9 7 , 2 0}$ & $\mathbf{9 7 , 2 2}$ & $\mathbf{0 , 9 4 4 3}$ \\
\hline Sem segmentação & & & & \\
\hline AlexNet & 97,20 & 97,20 & 97,22 & 0,9439 \\
CaffeNet & 94,70 & 94,40 & 94,44 & 0,8871 \\
VggNet & 98,20 & 98,10 & 98,14 & 0,9622 \\
AlexNet + CaffeNet + VggNet & $\mathbf{9 8 , 2 0}$ & $\mathbf{9 8 , 1 0}$ & $\mathbf{9 8 , 1 4}$ & $\mathbf{0 , 9 6 2 5}$ \\
\hline
\end{tabular}

deve a outros elementos existentes na imagem, como o citoplasma, as hemácias e o fundo. Com a presença destes, a rede consegue assimilar mais informações relevantes durante a extração de características e consequentemente auxiliando na alta taxa de acerto.

Os trabalhos da literatura que lidam com sistemas de classificação de leucemia com mais de um núcleo, evidenciam que muitos trabalhos buscam classificar apenas as lâminas com um núcleo, enquanto a classificação com vários núcleos é ignorada. No nosso trabalho, comparamos os resultados obtidos pelo sistema proposto com outros trabalhos que buscam realizar esse tipo de classificação (Tabela 2).

Tabela 2. Comparação da acurácia com outros métodos da literatura (ALL-IDB1).

\begin{tabular}{|c|c|c|}
\hline Trabalho & Quantidade de imagens & A(\%) \\
\hline [Madhukar et al. 2012] & 98 & 93,50 \\
[Vincent et al. 2015] & 100 & 97,70 \\
[Patel and Mishra 2015] & 27 & 93,75 \\
[Agaian et al. 2016] & 98 & $94,00 *$ \\
Método proposto & 108 & $\mathbf{9 8 , 1 4}$ \\
\hline
\end{tabular}

No trabalho proposto por Agaian et al. [Agaian et al. 2016], os autores não apresentam o valor exato da acurácia do seu sistema, apenas enfatizam que foi superior a 94\% de acerto. Em outro com os mesmos autores ([Madhukar et al. 2012]), o resultado obtido foi de $93,50 \%$, sendo utilizada a mesma base de imagens. O trabalho de Patel e Mishra [Patel and Mishra 2015] atingiu 93,75\% de acurácia, sendo superior ao de Madhukar et al. [Madhukar et al. 2012], no entanto, a menor quantidade de imagens torna o sistema não tão confiável se comparado com outros trabalhos com uma base maior. Já o sistema descrito neste trabalho utiliza uma quantidade de imagens maior que os outros, proporcionando uma maior confiabilidade nos resultados e apresenta a acurácia mais alta dentre estes, com 98,17\%, validando assim a abordagem para a base ALL-IDB1.

$\mathrm{Na}$ construção do comitê, diversos classificadores foram testados empiricamente e dentre estes, três foram escolhidos por apresentarem melhores resultados. Acreditase que as taxas obtidas podem ser melhoradas, uma vez que o método de transferência de aprendizado não consegue assimilar completamente as características das imagens de sangue, já que as redes foram treinadas para extrair características genéricas e não específicas da doença. Dentre os três modelos de arquiteturas utilizados, a AlexNet e a VggNet atingiram valores próximos, sendo que este resultado indica que redes mais rasas, como 
AlexNet, aprendem características mais gerais que são aplicáveis a uma variedade mais ampla de imagens. Os recursos aprendidos da AlexNet são menos otimizados semanticamente para imagens naturais, são mais generalizáveis e adaptáveis quando transferidos para o domínio da imagem médica. A abordagem proposta utilizando apenas a saída da VggNet obteve resultados semelhantes a saída concatenada, observando que esta sem a etapa de segmentação atingiu resultados iguais. Realizado um estudo na relevância das características do vetor concatenado, observou-se que os atributos provenientes da VggNet são os que possuem o maior ganho de informação. No entanto, deve-se atentar que nem sempre os atributos desta rede terão esse ganho considerável.

\section{Conclusão}

Como a extração de características e a classificação são considerados os passos mais importantes no diagnóstico automático de diferentes sistemas computacionais, constatou-se que diversos métodos da literatura apresentaram resultados promissores. No entanto, necessitam de maior robustez e de uma maior quantidade de imagens para obter uma melhor desempenho e confiabilidade nos seus resultados. O trabalho apresentado neste artigo descreve um novo sistema para o diagnóstico de leucemia em imagens de sangue com a utilização das Redes Neurais Convolucionais e de um comitê com três classificadores. Baseando-se nos resultados obtidos pela abordagem, é possível validar a sua robustez perante outros trabalhos do estado da arte com uma acurácia de 98,14\%.

Como trabalhos futuros, propõe-se a utilização de um ajuste fino na rede, para que seja possível melhorar a abstração de informações da leucemia. Apesar da abordagem atual não requerer uma grande quantidade de processamento, ela ainda não representa $o$ poder máximo das CNN's. Além do melhoramento do sistema, pretende-se utilizar novas bases de imagens, com uma maior quantidade de dados, para que esse sistema possa ser validado e utilizado no cotidiano, ajudando os médicos e os pacientes no diagnóstico dessa doença.

\section{Referências}

Agaian, S., Madhukar, M., and Chronopoulos, A. T. (2016). A new acute leukaemiaautomated classification system. Computer Methods in Biomechanics and Biomedical Engineering: Imaging \& Visualization, pages 1-12.

Castelluccio, M., Poggi, G., Sansone, C., and Verdoliva, L. (2015). Land use classification in remote sensing images by convolutional neural networks. CoRR, abs/1508.00092.

Chatfield, K., Simonyan, K., Vedaldi, A., and Zisserman, A. (2014). Return of the devil in the details: Delving deep into convolutional nets. In British Machine Vision Conference.

Chen, X., Xu, Y., Wong, D. W. K., Wong, T. Y., and Liu, J. (2015). Glaucoma detection based on deep convolutional neural network. In 2015 37th Annual International Conference of the IEEE Engineering in Medicine and Biology Society (EMBC), volume 37, pages 715-718.

Jia, Y., Shelhamer, E., Donahue, J., Karayev, S., Long, J., Girshick, R., Guadarrama, S., and Darrell, T. (2014). Caffe: Convolutional architecture for fast feature embedding. In Proceedings of the 22Nd ACM International Conference on Multimedia, MM '14, pages 675-678, New York, NY, USA. ACM. 
Jr., M. P. P. (2011). Combining classifiers: From the creation of ensembles to the decision fusion. In SIBGRAPI Tutorials, pages 1-10. IEEE Computer Society.

Kawahara, J. and Hamarneh, G. (2016). Multi-resolution-Tract CNN with Hybrid Pretrained and Skin-Lesion Trained Layers. Springer International Publishing, Cham.

Krizhevsky, A., Sutskever, I., and Hinton, G. E. (2012). Imagenet classification with deep convolutional neural networks. In Pereira, F., Burges, C. J. C., Bottou, L., and Weinberger, K. Q., editors, Advances in Neural Information Processing Systems 25, pages 1097-1105. Curran Associates, Inc.

Kumar, A., Kim, J., Lyndon, D., Fulham, M., and Feng, D. (2016). An ensemble of finetuned convolutional neural networks for medical image classification. IEEE Journal of Biomedical and Health Informatics, PP:1-9.

Labati, R. D., Piuri, V., and Scotti, F. (2011). All-idb: The acute lymphoblastic leukemia image database for image processing. Image Processing (ICIP), 2011 18th IEEE International Conference on, pages 2045-2048.

Madhukar, M., Agaian, S., and Chronopoulos, A. T. (2012). New decision support tool for acute lymphoblastic leukemia classification. Image Processing: Algorithms and Systems X; and Parallel Processing for Imaging Applications II, 8295.

Madhukar, M., Agaian, S., and Chronopoulos, A. T. (2014). Automated screening system for acute myelogenous leukemia detection in blood microscopic images. IEEE Systems Journal, 8(3).

Mohapatra, S., Patra, D., and Satpathy, S. (2014). An ensemble classifier system for early diagnosis of acute lymphoblastic leukemia in blood microscopic images. Neural Computing and Applications, 24:1887-1904.

Patel, N. and Mishra, A. (2015). Automated leukaemia detection using microscopic images. 58:635-642.

Shin, H., Roth, H. R., Gao, M., Lu, L., Xu, Z., Nogues, I., Yao, J., Mollura, D. J., and Summers, R. M. (2016). Deep convolutional neural networks for computer-aided detection: $\mathrm{CNN}$ architectures, dataset characteristics and transfer learning. CoRR, abs/1602.03409.

Vincent, I., Kwon, K.-R., Lee, S.-H., and Moon, K.-S. (2015). Acute lymphoid leukemia classification using two-step neural network classifier. Frontiers of Computer Vision $(F C V)$, pages $1-4$.

Vogado, L. H. S., de M S Veras, R., Andrade, A. R., e Silva, R. R. V., de Araujo, F. H. D., and de Medeiros, F. N. S. (2016). Unsupervised leukemia cells segmentation based on multi-space color channels. In International Symposium on Multimedia, pages 451456. IEEE.

Wang, D., Khosla, A., Gargeya, R., Irshad, H., and Beck, A. H. (2016). Deep Learning for Identifying Metastatic Breast Cancer. ArXiv e-prints.

Zack, G. W., Rogers, W. E., and Latt, S. A. (1977). Automatic measurement of sister chromatid exchange frequency. Journal of Histochemistry \& Cytochemistry, pages 741-753. 